\title{
Suppressor of cytokine signalling protein SOCS3 expression is increased at sites of acute and chronic inflammation
}

\author{
Gemma E. White $\cdot$ Andrew Cotterill • \\ Mark R. Addley $\cdot$ Elizabeth J. Soilleux . \\ David R. Greaves
}

Received: 18 September 2010/ Accepted: 26 January 2011/Published online: 1 March 2011

(C) The Author(s) 2011. This article is published with open access at Springerlink.com

\begin{abstract}
Treatment of cells with cytokines and growth factors leads to the synthesis of Suppressor of Cytokine Signalling (SOCS) proteins that act as potent negative regulators of signalling via the Jak/STAT pathway. We used immunohistochemistry to identify cells and pathologies where SOCS3 expression might influence acute and chronic inflammatory responses in human tissues. Epitope and GFP tagged SOCS3 fusion proteins were localised predominantly in the nucleus of transfected cells and a validated anti SOCS3 antiserum revealed the expression of SOCS3 in the nucleus and cytoplasm of macrophages, endothelial and epithelial cells in a wide range of normal tissues in tissue microarrays $(n=31$ different tissues). Nuclear SOCS3 was only seen in cells expressing a high level of the protein. Comparative immunostaining of acute, chronically and granulomatously inflamed human tissues revealed higher levels of nuclear and cytoplasmic SOCS3 expression in inflamed than in corresponding normal tissues, particularly in recruited leukocyte populations, but also in epithelia. The staining appeared more intense, suggesting higher expression levels, in areas where
\end{abstract}

G. E. White and A. Cotterill contributed equally to the study. E. J. Soilleux and D. R. Greaves contributed equally to the study.

G. E. White - M. R. Addley · D. R. Greaves

Sir William Dunn School of Pathology, University of Oxford, South Parks Road, Oxford OX1 3RE, UK

A. Cotterill · E. J. Soilleux

Department of Cellular Pathology, John Radcliffe Hospital,

Oxford OX3 9DU, UK

E. J. Soilleux $(\bowtie)$

Nuffield Department of Clinical Laboratory Sciences, John Radcliffe Hospital, University of Oxford, Oxford OX3 9DU, UK

e-mail: Elizabeth.Soilleux@ndcls.ox.ac.uk inflammation was more acute, consistent with the time course of SOCS3 induction described in vitro. Expression of SOCS3 protein by leucocytes and other cell types in tissue sections could be a useful marker of cells undergoing acute or chronic stimulation by cytokines in vivo.

Keywords SOCS3 - Transcription factor - Expression · Granuloma $\cdot$ Macrophage $\cdot$ Tissue $\cdot$ Inflammation

\section{Introduction}

The inflammatory response is driven by the release of inflammatory mediators and the recruitment of circulating leukocytes, which become activated at the site of inflammation and release further mediators. Generally, the inflammatory response is resolved by the release of endogenous anti-inflammatory mediators and the accumulation of intracellular negative regulatory factors (Nathan 2002). Failure to regulate inflammation adequately is important in autoimmunity and infection and may play a role in chronic diseases such as coronary artery disease, obesity and cancer (Lin and Karin 2007). Anti-cytokine therapies, such as anti-tumor necrosis factor- $\alpha$, can successfully treat some chronic inflammatory diseases (Feldmann and Maini 2003).

Suppressor of cytokine signalling (SOCS) proteins are intracellular inhibitors of cytokine signalling that act in a classical negative feedback loop (Croker et al. 2004). SOCS3 may have profound effects on the regulation of immunity and inflammation by affecting the activation, development and homeostatic functions of all lineages involved in immune and inflammatory responses (O'Shea and Murray 2008). For example, SOCS3 limits the activities of the haematopoietic growth factors granulocyte 
colony stimulating factor (G-CSF) and granulocyte-macrophage colony stimulating factor (GM-CSF) (Croker et al. 2004). Administration of G-CSF in vivo mimics emergency granulopoiesis during infection. In SOCS3 conditional knockout mice, this process is grossly exacerbated, with neutrophil infiltration and destruction of liver, lung, muscle and spinal tissue, resulting from increased intensity and duration of G-CSF-induced Stat3 activation (Croker et al. 2003; Kimura et al. 2004).

Stimulation of macrophages with lipopolysaccharide (LPS) leads to the production of various cytokines including IL-1, TGF- $\beta$, IL-10, and IL-6, all of which upregulate SOCS3 expression (Dimitriou et al. 2008). Tolllike receptor (TLR) stimulation can also upregulate SOCS3 expression independently of cytokine induction (Dalpke et al. 2008). Indeed SOCS3 is one of the most abundantly induced proteins in LPS-stimulated macrophages (Yoshimura et al. 2007). SOCS3 then mediates feedback inhibition of LPS-induced macrophage activation (Berlato et al. 2002; Donnelly et al. 1999). SOCS3-transfected murine macrophages produce reduced levels of LPSinduced $\mathrm{NO}_{2}{ }^{-}$, TNF- $\alpha$, IL- 6 , and GM-CSF. The extent of this inhibition is similar to that seen in wild type cells treated with LPS and IL-10 (Berlato et al. 2002; Dimitriou et al. 2008). Accordingly, primary peritoneal SOCS3-/macrophages exhibit impaired IL-10 regulation of LPS-induced $\mathrm{NO}_{2}{ }^{-}$and TNF- $\alpha$ (Berlato et al. 2002). Macrophages from mice that lack SOCS3 expression in all haematopoietic and endothelial lineages produce more IL-6 following IL-1 stimulation (Wong et al. 2006). SOCS3 mRNA is induced rapidly following cytokine stimulation, being detectable within 20 min of IL- 6 stimulation and declining to basal levels after $8 \mathrm{~h}$ (Larsen and Ropke 2002). Notwithstanding, SOCS3 has also been shown to positively regulate TLR4 signalling and M1 polarisation of macrophages in mouse and rat (Wang et al. 2010; Liu et al. 2008a, b).

SOCS1 and SOCS3 exert their negative feedback via several distinct mechanisms: (1) blocking JAK catalytic domain-STAT protein substrate interactions to terminate signal propagation; (2) competitively inhibiting JAK and STAT binding to phosphotyrosine-based docking sites on activated cytokine receptors, such as the shared cytokine subunit gp130; (3) targeting their binding partners for ubiquitination and proteasome degradation (Dimitriou et al. 2008). The SOCS3 protein inhibits signal transduction via various receptors, including the IL-6R, IFN- $\gamma$ R, GM-CSFR, IL-12 receptor $\beta 2$, erythropoietin receptor, IL-27R $\alpha$, IL-31R $\alpha$, G-CSF receptor, and leptin receptor (Dimitriou et al. 2008; Dalpke et al. 2008; Xu et al. 2010).

However, SOCS3 does not bind or inhibit signalling via the IL-10R, explaining the divergent activities of the proinflammatory cytokine IL-6 and the immunoregulatory cytokine IL-10 following TLR stimulation (Dimitriou et al. 2008; Yoshimura et al. 2007). Induction of SOCS has been suggested as a means of immune evasion for bacteria, viruses and parasites, for example Mycobacterium bovis BCG (Imai et al. 2003; Dalpke et al. 2008). SOCS3 expression in dendritic cells (DCs) is important in regulating the production of cytokines able to direct naïve $\mathrm{T}$-cell differentiation towards a $\mathrm{T}_{\mathrm{h} 2}$ or a $\mathrm{T}_{\text {reg }}$ phenotype (Li et al. 2006; Dimitriou et al. 2008). Naïve $T_{h}$ cells express high levels of SOCS3, which are transiently decreased after antigenic stimulation, allowing increased proliferation in response to IL-2. Both unstimulated and stimulated $\mathrm{T}_{\text {reg }}$ cells exhibit low SOCS3 levels (Dimitriou et al. 2008). A negative regulatory role for SOCS3 in IL-1 signalling has been observed in B-cells, in which SOCS3 negatively regulates IL-1R signalling pathways (Dimitriou et al. 2008).

SOCS3 knockout leads to placental defects, possible disturbances in intrahepatic erythropoiesis and embryonic lethality (Roberts et al. 2001; Marine et al. 1999; Larsen and Ropke 2002). Mice with a conditional deletion of the Socs 3 gene in haematopoietic and endothelial cells die as young adults due to severe inflammatory lesions in the peritoneal and pleural cavities (Croker et al. 2008). Transgenic overexpression of SOCS3 also leads to embryonic lethality, while SOCS3 overexpression in T-cells leads to increased Th2 differentiation (Dalpke et al. 2008). SOCS3 mRNA expression has been identified in foetal liver, primarily during the stage of fetal liver erythropoiesis (Marine et al. 1999), and, at low levels, in adult mouse spleen, thymus and lung (Starr et al. 1997). A limited immunohistochemical study demonstrated SOCS3 expression in colonic epithelium and lamina propria leucocytes in biopsies from normal individuals and ulcerative colitis patients ( $\mathrm{Li}$ et al. 2010).

The intracellular localisation of SOCS proteins has been controversial, with most authors reporting only a cytoplasmic localisation (Ding et al. 2003), although Lee and colleagues observed cytoplasmic and nuclear localisation (Lee et al. 2008). Nuclear localisation, however, seems inconsistent with SOCS proteins mediating their effects solely at the cell membrane (Dimitriou et al. 2008; Ding et al. 2003). To date detailed analysis of the expression pattern and intracellular localisation of SOCS proteins in human tissue samples has been limited by the availability of specific anti-human SOCS3 antisera that work on formalin fixed tissue.

Here we report the subcellular localisation of SOCS3 fusion proteins in transfected cells and we validate an antiSOCS3 polyclonal rabbit serum and use it to determine the expression pattern of SOCS3 protein in a wide range of human tissues under normal and inflammatory conditions. By means of immunohistochemistry (IHC), we demonstrate both cytoplasmic and nuclear expression of SOCS3 
in vitro and in vivo. SOCS3 expression in tissue samples may be a useful marker of cells undergoing acute and, to a lesser extent, chronic cytokine stimulation in vivo.

\section{Materials and methods}

\section{SOCS1 and SOCS3 fusion proteins}

Human SOCS1 and SOCS3 coding sequences were amplified by PCR and cloned into the expression plasmid pcDNA3.1, together with the GFP gene. The coding region of the recombinant plasmids was confirmed by DNA sequencing.

\section{IL-4 luciferase assay}

Murine TARC luciferase reporter plasmids, SOCS expression vectors and hCMV $\beta$-galactosidase plasmids were co-transfected into sub-confluent $\mathrm{CHO}$ cells using Genejuice $^{\circledR}$ (Merck) in serum free Optimem medium (InVitrogen) (Liddiard et al. 2006). After $18 \mathrm{~h}$, cells were stimulated with $20 \mathrm{ng} \mathrm{ml}^{-1}$ Interleukin-4 (R\&D Systems) for $6 \mathrm{~h}$. Whole cell extracts were prepared in Reporter Lysis Buffer (Promega) and luciferase enzyme activity was determined using the Promega luciferase assay kit and $\beta$-galactosidase enzyme activity in the same lysates was assayed by measuring conversion of the colorimetric substrate chlorophenol red- $\beta$-D-galactopyranoside (Boeringer Mannheim) at $550 \mathrm{~nm}$ as described previously (Liddiard et al. 2006).

\section{Preparation of transfectants and western blot}

HEK293T cells were seeded into a six well plate and transfected with GFP, SOCS2 or SOCS3 expression plasmids using Genejuice ${ }^{\circledR}$ (Merck). Total cell lysates were prepared $36 \mathrm{~h}$ post transfection and western blots performed. Total protein $(40 \mu \mathrm{g})$ was resolved on an SDSPAGE gel and western blotted onto nitrocellulose (in duplicate). The blot was incubated with rabbit polyclonal anti-SOCS3 sera (ab16030 and ab53984) (ABCAM, Cambridge, UK) and bands detected with goat anti-rabbit horseradish peroxidase (HRP) (Biorad) and chemiluminescence (SuperSignal West Pico, Pierce). The blot was stripped and re-probed with rabbit anti-tubulin polyclonal antiserum (Cell Signaling Technology) to demonstrate equal protein loading in all lanes.

Preparation of transfected paraffin wax cell blocks

Cell blocks of HEK293 cells transiently transfected with SOCS2, SOCS3 or GFP were prepared using the Shandon Cytoblock $^{\circledR}$ Cell Preparation System (Thermo Fisher
Scientific, Massachusetts, USA) as per the manufacturer's instructions and fixed in $4 \%$ formalin, followed by processing following standard histological techniques.

Lipopolysaccharide stimulation and immunofluorescent staining of human peripheral blood mononuclear cells

Human PBMCs were isolated from buffy coat preparations (National Blood Service, London, UK) using Ficoll-Paque plus according to manufacturer's instructions (GE Healthcare, Little Chalfont, UK). Cells were plated in 8 well LabTek chamber slides (Nunc, Rochester NY) and cultured in X-Vivo 10 medium (Lonza, Wokingham, UK) plus 1\% autologous serum for 7 days. Cells were stimulated for $4 \mathrm{~h}$ with $100 \mathrm{ng} / \mathrm{ml}$ LPS from Escherichia coli strain 055:B5 (Sigma-Aldrich, Gillingham, UK). After $4 \mathrm{~h}$, medium was removed, cells were fixed in $4 \%$ paraformaldehyde for $15 \mathrm{~min}$ then permeabilised for $5 \mathrm{~min}$ in $0.25 \%$ Triton X-100/PBS. Non-specific binding was blocked for $30 \mathrm{~min}$ with blocking solution (PBS/0.1\% Triton X-100/1\% BSA/ $200 \mu \mathrm{g} / \mathrm{ml}$ human IgG). Anti-SOCS3 antibody (1:50 dilution, ab53984, Abcam) or isotype control (rabbit IgG, Jackson Immunoresearch) was added and cells stained overnight at $4^{\circ} \mathrm{C}$. PE conjugated secondary antibody $(1: 200$ dilution, goat anti-rabbit IgG, Jackson Immunoresearch) was added for $2 \mathrm{~h}$ at room temperature. Nuclei were counterstained for $5 \mathrm{~min}$ with DAPI (Sigma-Aldrich). Slides mounted with fluorescent mounting medium (Sigma-Aldrich). Images were captured using a Zeiss 510 META confocal microscope.

Histological samples

All tissue samples were obtained with full ethical approval from the National Research and Ethics Service (REC reference 04/Q1604/21). A wide range of normal human tissues $(n=31)$ were obtained from the Cellular Pathology Department, John Radcliffe Hospital, Oxford, UK, and used to construct tissue microarrays (core diameter $1 \mathrm{~mm}$ ), using the MTA-1 manual arrayer (Beecher Instruments, Wisconsin, USA). Two cores from each donor block were taken to increase the sample area. Whole sections of tissue were similarly obtained.

Antibodies and immunostaining

The following primary antibodies were used: rabbit polyclonal against SOCS3 (ab53984, ABCAM, Cambridge, UK) and myeloperoxidase (AO398, Dako, Ely, UK); mouse monoclonal antibodies against DC-SIGN (clone DC28, R\&D Systems, Minneapolis, USA); CD3 (clone LN10) and CD56 (clone 1B6, Leica Biosystems Newcastle Ltd, Newcastle, UK); mouse monoclonal antibodies against CD20 
(clone L26), CD31 (clone JC70), CD61 (Y2/51), CD68 (clone PGM1) and glycophorin C (clone ret40f) were kindly donated by Prof. K. Gatter, Nuffield Department of Clinical Laboratory Sciences, Oxford, UK. Immunostaining was performed using standard techniques and a Bond $\operatorname{Max}^{\mathrm{TM}}$ immunostaining machine (Leica Biosystems Newcastle Ltd, Newcastle, U.K.). Heat mediated antigen retrieval was performed on all tissue sections using the high $\mathrm{pH}$ Epitope Retrieval 2 solution (Leica Microsystems, Wetzlar, Germany) for $20 \mathrm{~min}$ at $100^{\circ} \mathrm{C}$. This was followed by a $5 \mathrm{~min}$ peroxidase block as per the manufacturers' kit and instructions (Leica Microsystems, Wetzlar, Germany). For single labelled immunohistochemisty, the peroxidase/DAB Bond $^{\mathrm{TM}}$ Polymer Refine Detection System (Leica Microsystems, Wetzlar, Germany) was used for visualisation. For double labelled immunohistochemisty, the peroxidase/DAB Bond $^{\mathrm{TM}}$ Polymer Refine Detection System and alkaline phosphatase/fast red Bond ${ }^{\mathrm{TM}}$ Polymer AP Red Detection System (Leica Microsystems, Wetzlar, Germany) were used for visualisation. All slides were counterstained using Mayer's haematoxylin and mounted in DePeX (VWR International, Lutterworth, UK). As a negative control for the anti-SOCS3 antiserum, a section was immunostained with normal rabbit serum at an equivalent concentration $(0.0013 \mathrm{mg} / \mathrm{ml})$.

\section{Cell counting}

Four $20 \times$ microscope fields were selected for counting for each tissue sample. Three full sections of each condition and corresponding normal tissue were used. The same areas were marked and counted on each slide.

\section{Statistical analysis}

The Minitab $15^{\circledR}$ statistics package (Minitab, State College, PA, USA) was used for all statistical analyses. Results from cell population counts were converted to proportions and recorded as a percentage e.g. percentage of $\mathrm{CD} 68^{+}$ cells expressing SOCS3. Analysis of correlation was performed using the non-parametric Spearman's rank correlation coefficient (r). This was achieved by performing a Pearson correlation coefficient on ranked data. To identify differences in population medians the Mann-Whitney U test was used.

\section{Results}

Antibody validation

To establish the specificity of the SOCS3 antibodies we performed western blots of GFP, SOCS2 and SOCS3 transfected cell lysates. Incubation with anti-SOCS3 ab53984 detected a single band of the correct molecular weight in SOCS3 transfected cell lysate and this band was not detected in lysates prepared from GFP or SOCS2 transfected cells (Fig. 1a, upper panel). Stripping the blots and re-probing with an anti-tubulin antibody demonstrated equal protein loading in each lane (Fig. 1a, middle panel). SOCS3 was not detected with a second commercially available SOCS3 antiserum (Fig. 1a, lower panel). Single labelled immunohistochemistry (IHC) using the antiSOCS3 antibody ab53984 demonstrated positive staining in the SOCS3 CHO cell transfectants in formalin fixed paraffin blocks but did not identify protein expression in the GFP or SOCS2 CHO cell transfectants (Fig. 1b), thus confirming the specificity of the antibody. Of note immunohistochemical staining showed that SOCS3 protein was detectable in both the nuclear and cytoplasmic compartments of transfected cells. To characterise the antiserum further, immunohistochemical staining of formalin fixed paraffin embedded human tonsil tissue was undertaken, using rabbit serum as a negative control. Positive cytoplasmic and nuclear staining of a range of mononuclear cells was seen with the anti-SOCS3 serum (Fig. 1c,upper panel), but not with normal rabbit serum (Fig. 1c, lower panel). Similarly, immunofluorescent staining of lipopolysaccharide (LPS) stimulated peripheral blood mononuclear cells showed strong, predominantly nuclear staining (Fig. 1d, upper panel), while no staining was seen with an isotype control antibody (Fig. 1d, lower panel).

Fig. 1 Detection of SOCS3 protein in transiently transfected cells. a Lysates prepared from HEK 293 cells transiently transfected with pcDNA3.1 or the indicated SOCS expression vectors were fractionated by SDS PAGE and transferred to HyBond membrane. AntiSOCS3 antisera (ab53984 and ab16030) were used for western blotting at $1 / 1000$ dilution and an anti-rabbit IgG HRP antibody was used at $1 / 2000$ dilution as secondary antibody. b Transiently transfected 293 cells were harvested and fixed with $4 \%$ formalin before embedding and sectioning for IHC with anti SOCS3 antiserum ab53984 as described in "Methods and materials", 293 cells were transfected with SOCS3, GPF, or SOCS2 (40× magnification). c Normal tonsil immunostained for SOCS3 using rabbit polyclonal antiserum ab53984 at a final concentration of $0.0013 \mathrm{mg} / \mathrm{ml}$ (upper panel) compared with a negative control of normal tonsil immunostained with normal rabbit serum at the same concentration (lower panel). Scale bar (lower right) pertains to both panels and represents $50 \mu \mathrm{m}$. Panel D: SOCS3 is expressed in both the cytoplasm and nucleus of primary human macrophages stimulated with lipopolysaccharide (LPS). PBMCs (cultured for seven days) were stimulated with $100 \mathrm{ng} / \mathrm{ml}$ LPS for $4 \mathrm{~h}$. Cells were fixed, permeabilised and stained with anti-SOCS3 antibody (upper panel) or isotype control (lower panel) followed by goat anti-rabbit PE-conjugated secondary antibody. Nuclei were counterstained with DAPI. Cells with compact, unstained nuclei are lymphocytes. Data are representative of two independent donors 


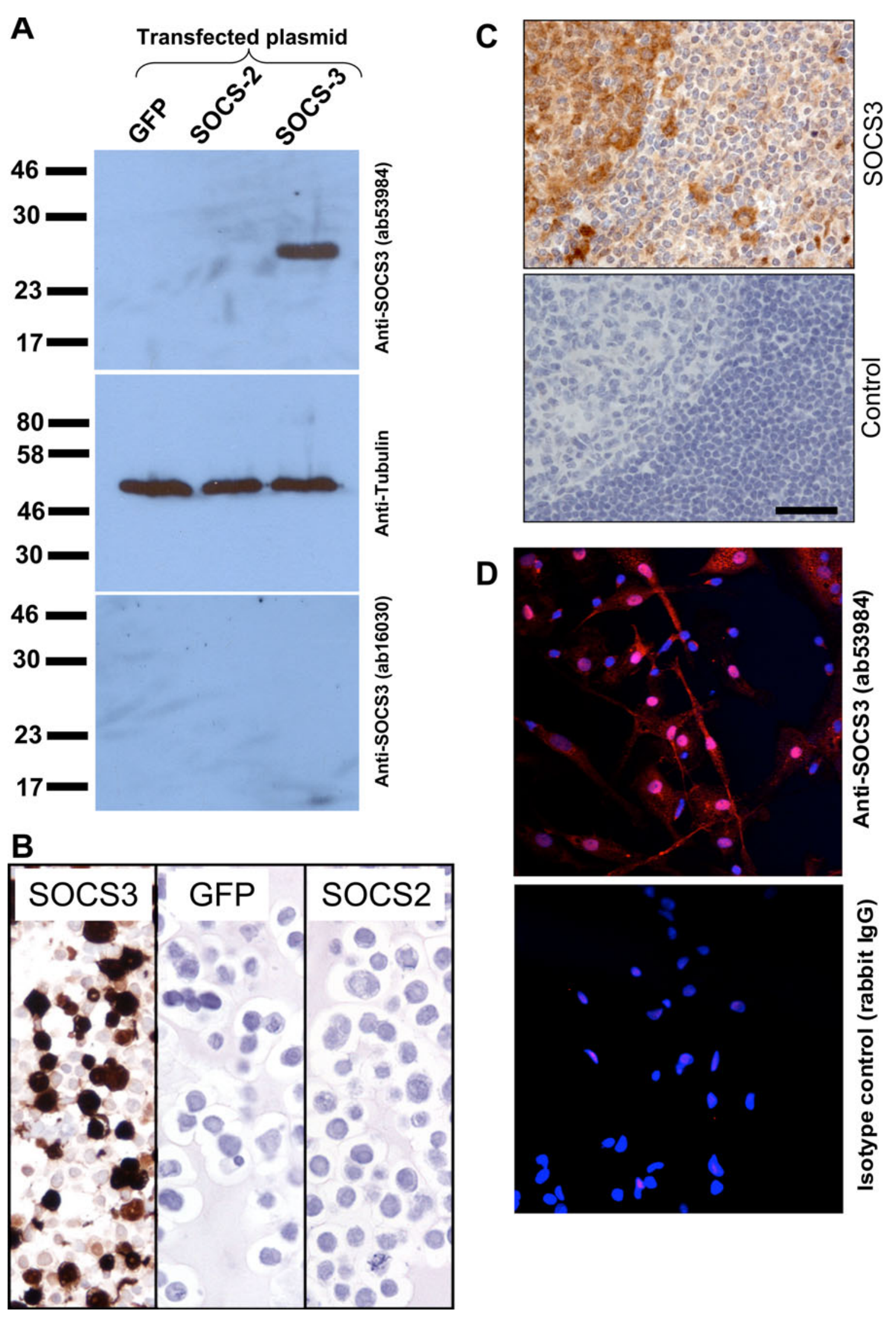


Sub-cellular localisation of SOCS1 and SOCS3

in transfected $\mathrm{CHO}$ cells

In order to study the sub-cellular localisation of SOCS3 in living cells we transiently transfected $\mathrm{CHO}$ cells with SOCS1-GFP and SOCS3-GFP fusion proteins and observed them using confocal microscopy. Figure 2 shows the distribution of GFP alone, SOCS1-GFP and SOCS3GFP in transfected CHO cells. GFP staining was distributed throughout the cell localising in both the nucleus and cytoplasm (Fig. 2a). SOCS1-GFP and SOCS3-GFP were localised predominantly in the nucleus, but were also present to a lesser extent in the cytoplasm (Fig. 2b, c). No fluorescence was detected in non-transfected cells (data not shown). We tested whether the presence of the GFP-tag would inhibit the ability of the SOCS protein to inhibit Jak/ STAT signalling using the IL-4 inducible murine TARC promoter in transiently transfected $\mathrm{CHO}$ cells. As shown in Fig. 3, the presence of the GFP tag does not affect the ability of the SOCS1 or SOCS3 proteins to inhibit IL-4 signalling. However, we cannot state categorically that the presence of the GFP-tag does not alter other properties of the SOCS proteins, including subcellular localisation.

\section{SOCS3 Immunohistochemistry on normal human tissue}

In normal human tissues, SOCS3 immunopositivity was widespread with varying intensity. Cells with lower levels of expression demonstrated exclusively cytoplasmic SOCS3 expression, while appreciable levels of nuclear SOCS3 were visible in cells with higher cytoplasmic expression levels. SOCS3 expression could be seen in endothelial cells, macrophages, DCs, lymphocytes, neutrophils and in various epithelial cells in a wide range of normal human tissues, including tonsil, skin, testis (Fig. 4a), cervix, endometrium, fallopian tube (Fig. 4b), kidney, small and large intestine and lung (Table 1, Fig. 4). Epithelia that did not express SOCS3 included salivary gland, oesophagus, stomach pancreas, liver, adrenal, parathyroid and ovary. None of these tissues contained constitutive lymphoid infiltrates or significant numbers of other leucocytes. SOCS3 protein showed an interesting distribution in lymphoid tissue, including spleen, lymph node, tonsil and the small and large intestinal lamina propria. Strong immunopositivity was seen in tingible body macrophages, interdigitating DCs and endothelial cells in sinuses and germinal centre B lymphocytes. B lymphocytes in the mantle and marginal zone showed little SOCS3 expression, as did paracortical $\mathrm{T}$ lymphocytes. In the thymus, cells mediating positive and negative selection, such as macrophages, DCs and epithelial cells, showed strong SOCS3 immunopositivity, while thymocytes were largely SOCS3-negative.
Fig. 2 Localisation of SOCS1 and SOCS3 in transfected CHO cells using confocal microscopy. Plasmids encoding GFP-alone (a), SOCS1-GFP (b), and SOCS3-GFP (c) were transiently transfected into $\mathrm{CHO}$ cells grown on glass coverslips in 24 well plates using GeneJuice. $24 \mathrm{~h}$ post-transfection the coverslips were removed, washed in PBS, fixed using 4\% paraformaldehyde and observed using confocal microscopy. Fluorescence images are on the left and phase contrast images on the right. Results are representative of three independent experiments

Double labelled immunohistochemistry confirmed SOCS3 expression in $\mathrm{CD}^{+} 8^{+}$macrophages/DCs (Fig. 4d), DC-SIGN $^{+}$DCs (Fig. 4e), and $\mathrm{CD} 31^{+}$endothelial cells (Fig. 4f). SOCS3 was also expressed by some CD3-positive T-lymphocytes, some CD20-positive B-lymphocytes and occasional CD56-positive natural killer cells in normal tissues (Table 1 and data not shown). The lymphocytes, macrophages, DCs and endothelial cells showed a very variable pattern of SOCS3 expression, ranging from no expression to strong expression with variable cytoplasmic/ nuclear distribution. In general, judging expression levels by immunohistochemical staining intensity, cells demonstrating nuclear expression of SOCS3 protein had higher cytoplasmic SOCS3 expression levels.

In bone marrow (Fig. 4c), strong SOCS3 immunostaining was seen in macrophages/DCs and in maturing cells of myeloid lineage with features of monocytic or granulocytic (neutrophil or eosinophil) differentiation, in addition to patchy weaker immunopositivity in some megakaryocytes and endothelial cells (Fig. 4). The identity of the cells was confirmed by double immunostaining for myeloperoxidase, glycophorin $\mathrm{C}$ and CD61 (data not shown). Cells of myeloid, macrophage and endothelial cell lineage expressed high levels of SOCS3 in both a cytoplasmic and nuclear location, while megakaryocytes expressed intermediate levels of SOCS3 with none being present in the nuclei. Cells of erythroid lineage expressed very little SOCS3 and showed no nuclear staining.

SOCS3 expression in inflamed human tissues

We examined SOCS3 expression in acute, chronic and granulomatous inflammatory conditions (Fig. 5, Table 2). SOCS3 expression was seen in the majority of leucocytes, particularly neutrophils, macrophages and lymphocytes, in inflammatory lesions, regardless of the phenotype of the inflammation. However, SOCS3 immunostaining appeared to be most intense in areas of acute inflammation, e.g., acute appendicitis (Fig. 5a), acutely inflamed radicular cysts, in crypt abscesses in ulcerative colitis (Fig. 5b), adjacent to deep fissuring ulcers and in endothelial cells and circulating neutrophils in Crohn's disease (Fig. 5c), and in the central but not the peripheral parts of granulomas in various conditions (Fig. 5d-f). In these conditions, the 


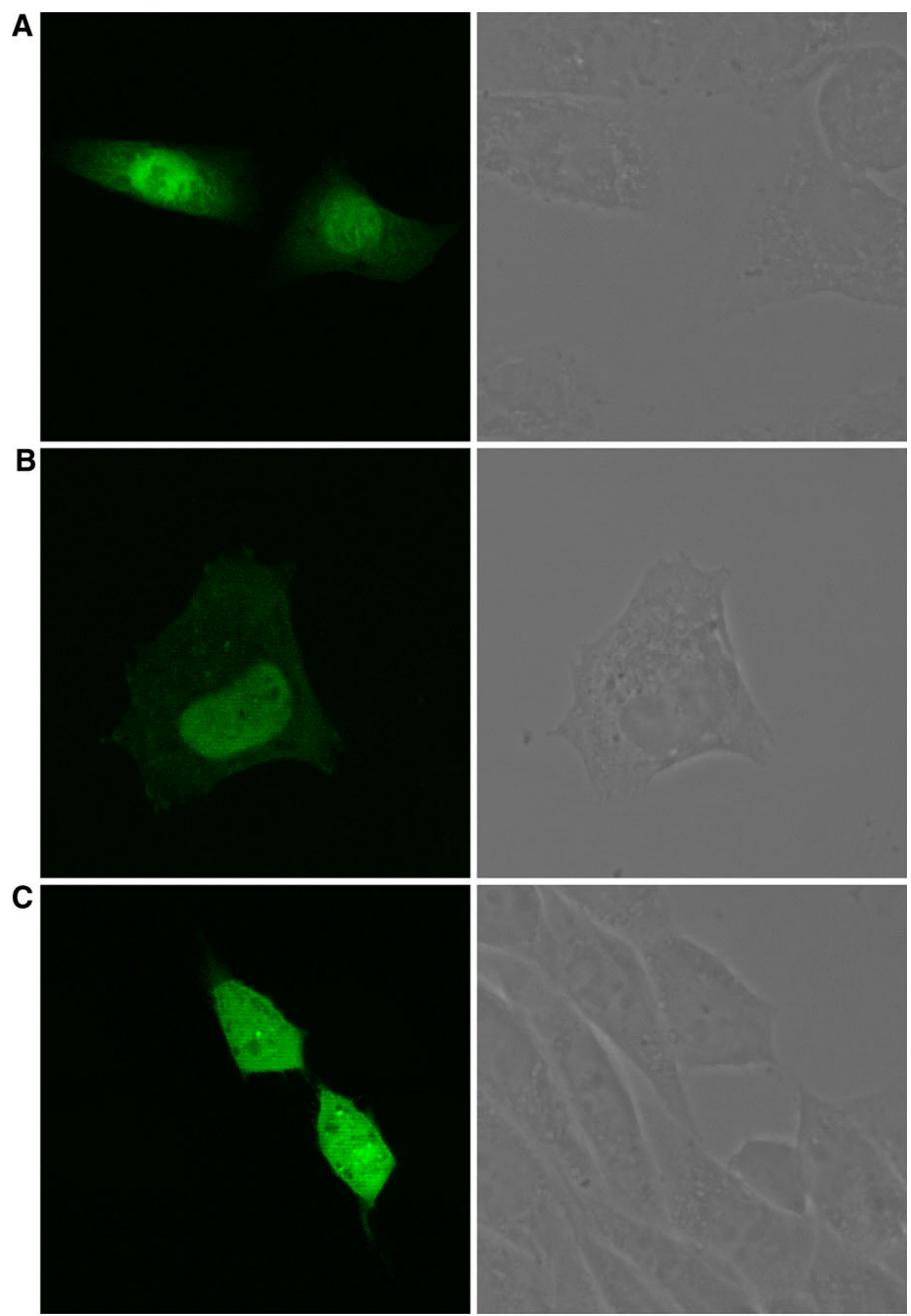




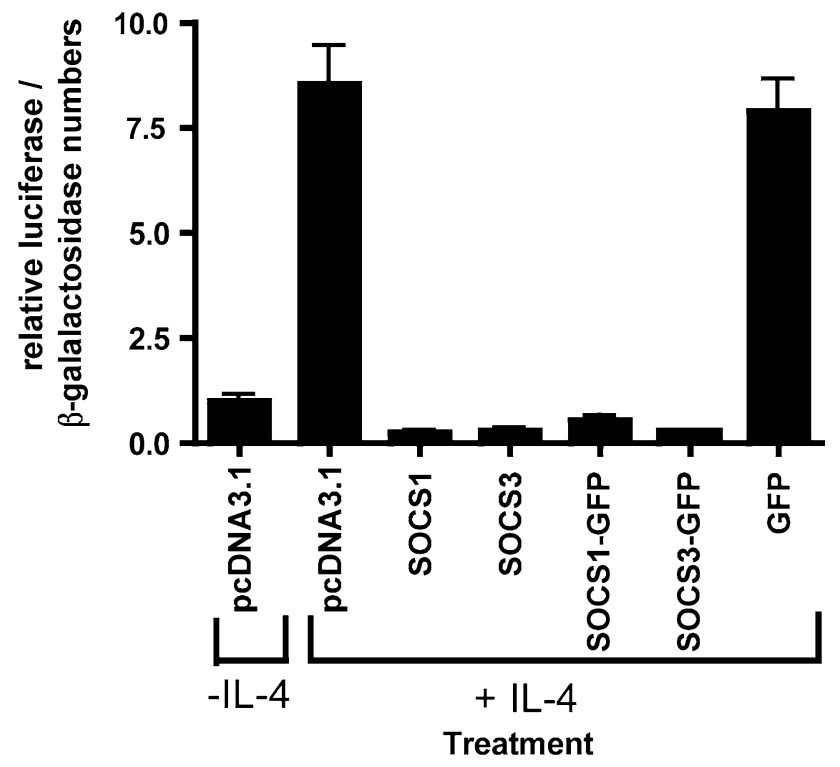

Fig. 3 TARC-luciferase assay in CHO cells showing that SOCS1GFP and SOCS3-GFP are functional. CHO cells $\left(2 \times 10^{6}\right)$ were transiently transfected with $500 \mathrm{ng}$ mTARC-luciferase, $100 \mathrm{ng}$ hCMV $\beta$-galactosidase, $200 \mathrm{ng}$ STAT6 and $200 \mathrm{ng}$ of the indicated SOCS expression vector. Cells were transiently transfected for $18 \mathrm{~h}$ then stimulated with IL-4 $\left(20 \mathrm{ng} \mathrm{ml}^{-1}\right)$ for $6 \mathrm{~h}$ before harvest in luciferase lysis buffer (Promega). Results are expressed as luciferase activity normalized for beta galactosidase activity and are representative of three independent experiments and data are presented as mean \pm SEM

cells expressing high levels of SOCS3 appeared to be predominantly neutrophils, together with a smaller number of macrophages and other leucocytes. A higher level of SOCS3 expression than that of the corresponding normal tissue could also be seen in some epithelial and endothelial cells adjacent to areas of intense acute inflammation, for example crypt abscesses of ulcerative colitis (Fig. 5b) and inflamed radicular cysts.

In order to investigate the effects of inflammation on expression of SOCS3 by macrophages and DCs, the percentage of $\mathrm{CD}^{+} 8^{+}$cells (macrophages/DCs) that expressed SOCS3 was determined in four $20 \times$ representative microscope fields in 3 cases of each of 7 pathological conditions with acute, chronic or granulomatous inflammation and compared with results for corresponding normal tissue. Each individual inflammatory condition demonstrated a higher percentage of $\mathrm{CD}^{+} 8^{+}$cells expressing SOCS3 than the corresponding normal tissue, with a significant $p$ value in the majority of cases (Table 2).

\section{Discussion}

In this study, we have used GFP-tagged forms of the human SOCS3 protein both to validate anti-SOCS3 antisera and investigate the subcellular localisation of SOCS3
Fig. 4 SOCS3 expression in normal human tissues. a-c show single immunostaining using the peroxidase technique (brown), while df show double immunostaining with SOCS3 detection by the peroxidase technique (brown) and detection of other antigens by the alkaline phosphatase technique (red). a Testicular tubules (TUB) showing strong cytoplasmic and weaker nuclear SOCS3 immunopositivity, together with weaker immunopositivity in adjacent macrophages (MAC). b Fallopian tube epithelium (EP) showing strong cytoplasmic and weaker nuclear SOCS-immunopositivity. c Bone marrow showing strong cytoplasmic and nuclear SOCS3 immunopositivity in cells of myeloid lineage (MYEL) including monocytes and neutrophils and their precursors, weak cytoplasmic immunopositivity in megakaryoctes (see Fig. 5h) and minimal immunopositivity in cells of erythroid lineage (ERY). d Small intestinal mucosa immunostained for SOCS3 (brown) and CD68 (red) demonstrating nuclear and cytoplasmic SOCS3 expression in some macrophages/ dendritic cells (arrows), in addition to SOCS3 immunopositivity in epithelium (EP) and a range of other leucocytes. e Lymph node immunostained for SOCS3 (brown) and DC-SIGN (red) demonstrating strong nuclear and weaker cytoplasmic SOCS3 immunopositivity (arrows) in interdigitating dendritic cells in the paracortex. Various lymphocytes and macrophages also show strong nuclear SOCS3 immunopositivity (arrowheads). f Duodenal submucosal vascular/ lymphatic channels demonstrating strong nuclear and cytoplasmic SOCS3 immunopositivity (brown, arrows) in CD31-positive (red) endothelial cells. Scale bars (bottom right of a-f) represent $50 \mathrm{~m}$. g. Magnified view of $\mathbf{b}$ to demonstrate cells with cytoplasmic staining only (arrowheads) compared with those with both nuclear and cytoplasmic staining (arrows). h Magnified view of $\mathbf{c}$ to demonstrate a megakaryocyte with cytoplasmic staining only (arrowhead) compared with myeloid cells showing both nuclear and cytoplasmic staining (arrows). Scale bars (bottom right of a-f) represent $15 \mathrm{~m}$. (Color figure online)

protein. We demonstrate, by means of transfection immunohistochemical studies and immunofluorescent staining of LPS-stimulated peripheral blood mononuclear cells, that SOCS3 protein may be present in both the nucleus and cytoplasm, in keeping with a previous study using SOCS3 transfectants (Lee et al. 2008). In vivo, we show that nuclear SOCS3 immunostaining is seen only in cells with high levels of cytoplasmic expression, consistent with in vitro data demonstrating SOCS3 nuclear translocation only when its levels increase (Lee et al. 2008). The transfectants used in our study are likely to overexpress SOCS3, explaining the observed, predominantly nuclear localisation of the GFP-tagged SOCS3 protein in these experiments. There has been one previous limited immunohistochemical study of SOCS3 expression in human intestine ( $\mathrm{Li}$ et al. 2010). While $\mathrm{Li}$ et al. describe cytoplasmic expression of SOCS3(Li et al. 2010), close examination of their immunohistochemical figures shows a predominantly cytoplasmic distribution in epithelial cells, but demonstrates a cytoplasmic and nuclear distribution of SOCS3 in leucocytes with morphological appearances of macrophages, DCs or lymphocytes in the large intestinal lamina propria, in agreement with our data.

Previous studies using mRNA analysis identified SOCS3 mRNA in normal mouse thymus, lung, spleen 


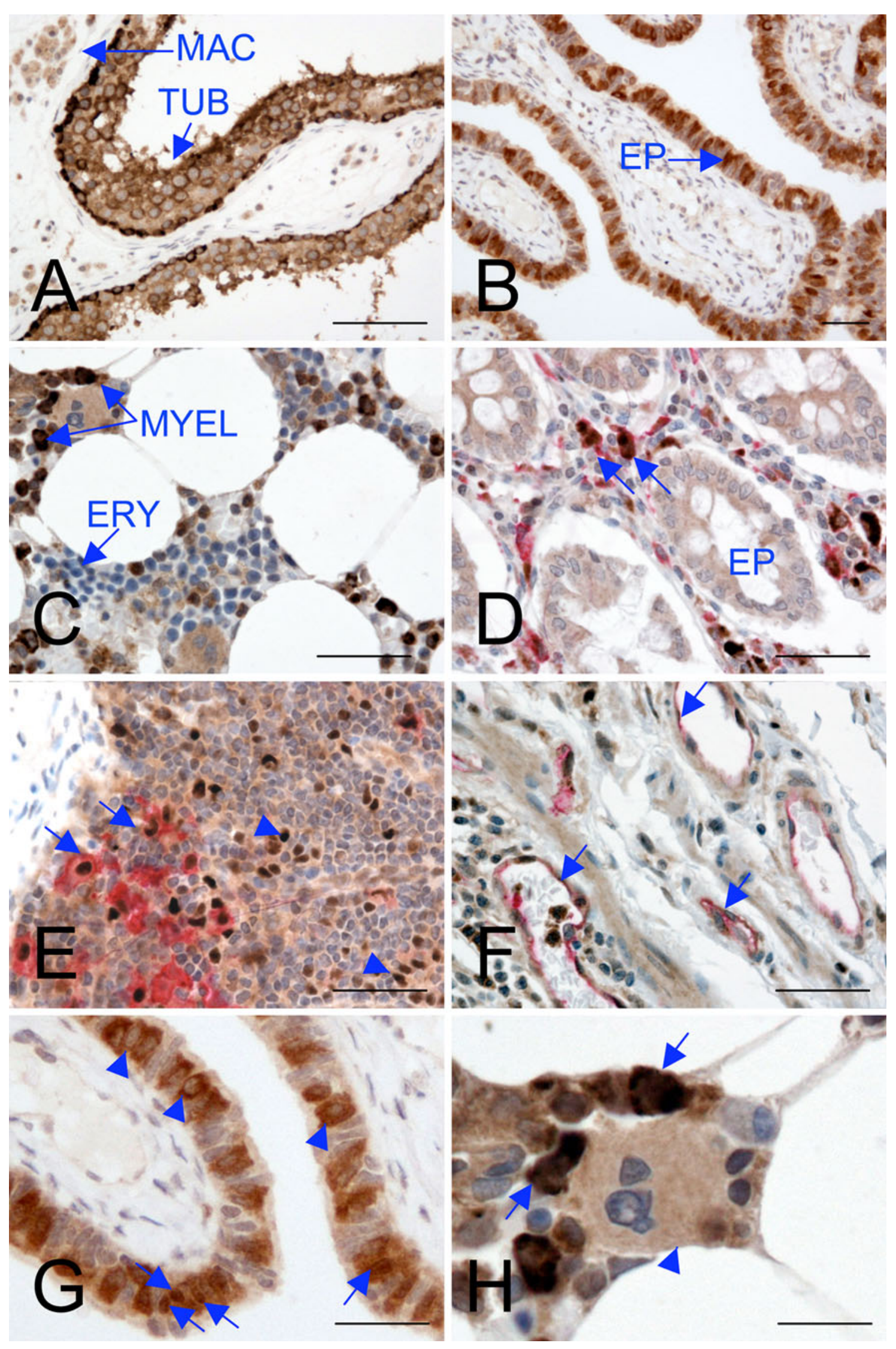




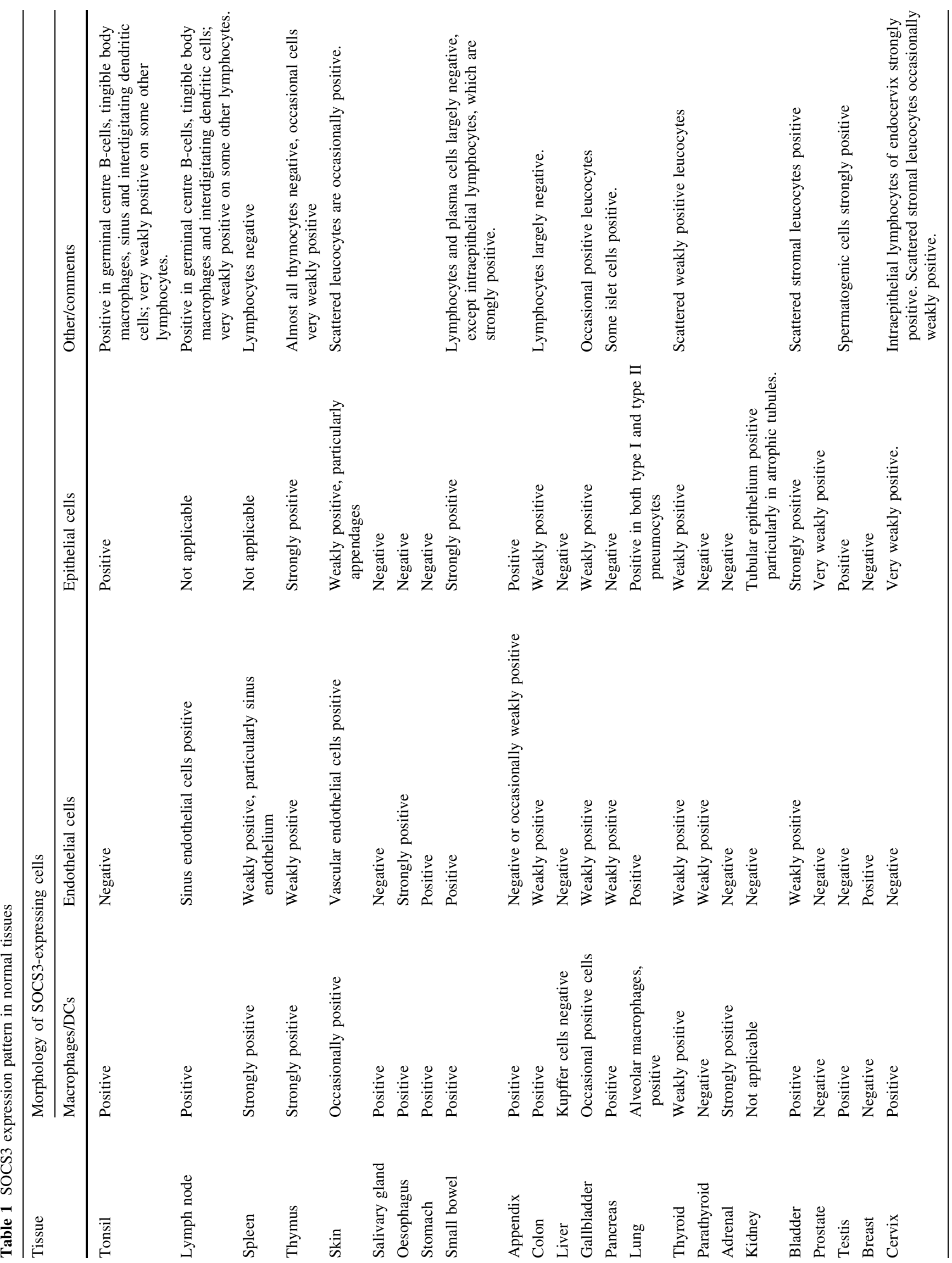




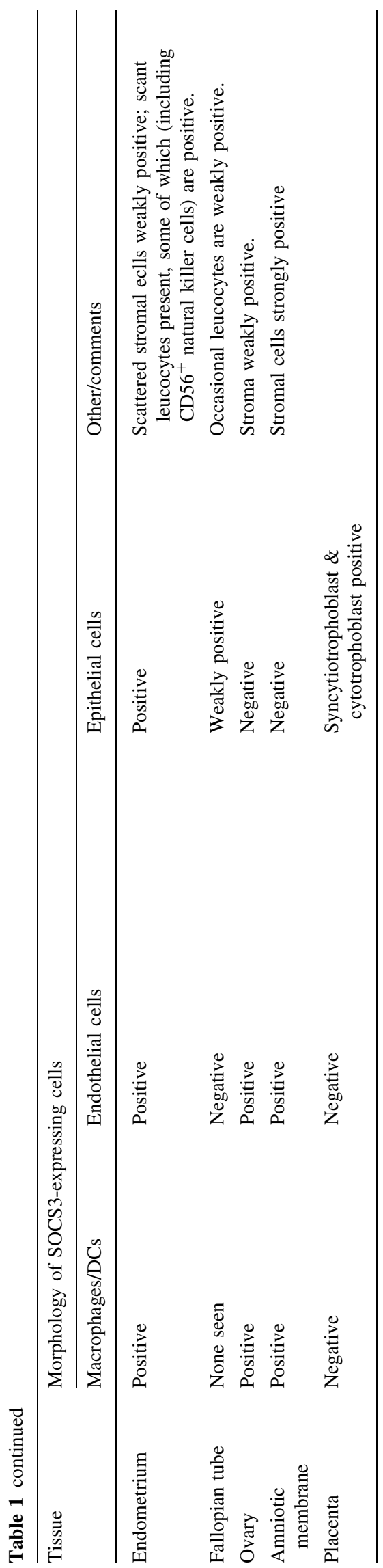

(Starr et al. 1997) and foetal liver (Marine et al. 1999), and in human T-cells, monocytes and macrophages in vitro (Isomaki et al. 2007; Rakshit et al. 2006). Using immunohistochemistry we were able not only to confirm the expression of SOCS3 protein in normal human adult thymus, lung, liver and spleen, but also to identify the major positive cell types as macrophages, immature myeloid DCs and endothelial cells. Additionally, in normal tissues, SOCS3 is expressed by neutrophils, some B and T-lymphocytes, occasional natural killer cells and many types of epithelium. Our demonstration of SOCS3 expression by epithelia at a number of sites is also in keeping with the study by $\mathrm{Li}$ et al. in which the authors show expression of SOCS3 by colonic epithelial cells ( $\mathrm{Li}$ et al. 2010). The figures in their study demonstrate SOCS3 expression by lamina propria leucocytes with morphological appearances suggesting that they might be macrophages, DCs or lymphocytes, although the authors do not comment on expression by these cell types or determine their identity. However, the SOCS3 expression pattern is consistent with the pattern we describe here, both in normal colon and in ulcerative colitis.

While epithelia adjacent to lymphoid aggregates or leucocytic infiltrates (e.g., small and large intestine, tonsil, cervix) express SOCS3, epithelia with few adjacent leucocytes are generally SOCS3 negative, e.g., salivary gland, oesophagus, stomach, pancreas and liver. Many epithelia express cytokine receptors, for example for TNF- $\alpha$, IL-1 and IL-6 (Fritz et al. 2008), which are known to induce SOCS3. Epithelia adjacent to lymphoid aggregates or leucocytic infiltrates may be subject to repeated stimulation by these cytokines, leading to the induction of SOCS3 expression detectable by IHC.

SOCS3 shows an interesting distribution in lymphoid tissue, including spleen, lymph node, tonsil and the small and large intestinal lamina propria. Strong immunopositivity is seen in tingible body macrophages, interdigitating DCs, DCs in sinuses and germinal centre B lymphocytes. Mantle/marginal zone B and paracortical $\mathrm{T}$ lymphocytes show little SOCS3 expression. Data from mouse studies indicate only very low levels of SOCS3 expression in progenitor B cells with a gradual increase in SOCS3 levels as B cells become more mature (Le et al. 2007). SOCS3 can inhibit focal adhesion kinase activation, preventing B cell adhesion to VCAM-1, increasing B-cell motility (Le et al. 2007). It is likely that germinal centre B-cells require significant motility to allow interaction with follicular DCs and T-cells. Mantle/marginal zone B-cells may remain in lymphoid areas for longer periods, thus requiring less motility and possibly explaining their lower levels of SOCS3 expression. An alternative explanation is discrepancies between the human and mouse immune systems in patterns of SOCS3 expression. Our observation of minimal 
expression of SOCS3 by paracortical T-lymphocytes appears incongruous with the observation in vitro that naïve $T_{h}$ cells express high levels of SOCS3, which are transiently decreased after antigenic stimulation, allowing increased proliferation in response to IL-2 (Dimitriou et al. 2008). One possibility is that the lymphoid tissue we examined contained predominantly $\mathrm{T}$ lymphocytes responding to antigen and thus undergoing activation/proliferation, with concomitantly decreased SOCS3 levels. Alternatively, there may be differences between the mouse immune system, in which much previous work has been done (Dimitriou et al. 2008), and the human immune system.

In the thymus, cells mediating positive and negative selection, such as macrophages, DCs and epithelial cells, showed high levels of SOCS3 expression. Little expression was seen in thymocytes, in keeping with a report showing that SOCS3 conditional knockout mice demonstrate normal T-cell development in the thymus, suggesting SOCS3 may not play a crucial role in thymocytes (Chen et al. 2006)

Cells expressing SOCS3 in normal tissues may be subject to cytokine stimulation. At sites where few cytokines are likely to be present, there may be some other, as yet undetermined mechanism of SOCS3 induction causing regulation of cytokine or TLR-mediated activation, possibly to prevent inappropriate induction of an inflammatory or immune response. Tissue macrophages or DCs may, for example, express SOCS3 to prevent them producing proinflammatory/immune cytokines and/or expressing crucial co-stimulatory molecules that might otherwise induce an immune or inflammatory response, with the inherent risks of breaking immunological tolerance or causing inflammatory damage (Hart 1997). Similarly, certain endothelia, particularly lymph node sinus endothelium and possibly some small blood vessel endothelium, may present antigen to lymphocytes to induce immunological tolerance (Soilleux et al. 2002). SOCS3 may be important at these sites in preventing the production of pro-inflammatory/immune cytokines. If such cells did present antigen to T lymphocytes, they might induce tolerance rather than immunity in these lymphocytes.

In bone marrow, cells of the granulocytic and monocytic lineage express high levels of SOCS3 (Fig. 4c), in keeping with data from SOCS3 conditional knockout mice demonstrating that SOCS3 is necessary to limit the activities of the haematopoietic growth factors G-CSF and GM-CSF (Croker et al. 2004; Croker et al. 2003; Kimura et al. 2004). Expression of SOCS3 by myeloid cells may also explain the appreciable levels of SOCS3 mRNA previously detected in foetal liver (Marine et al. 1999). Mutations in the tyrosine kinase JAK2 are identified in the majority of patients with myeloproliferative neoplasms, such as essential thrombocythaemia, a chronic proliferative neoplasm of megakaryocytes. JAK2, particularly when constitutively activated by mutation, phosphorylates a tyrosine residue in SOCS3 to inactivate it and escape its inhibition (Elliott et al. 2009; Hookham et al. 2007). Our demonstration for the first time of SOCS3 expression by normal megakaryocytes provides additional evidence for this mechanism.

In keeping with our data showing high levels of SOCS3 expression in LPS-stimulated peripheral blood mononuclear cells, particularly cells morphologically resembling macrophages, we observed a marked increase in SOCS3 expression in inflamed tissues, in many types of leucocyte, particularly neutrophils, macrophages and lymphocytes, as well as in epithelia, endothelia and fibroblasts. Given our observation of high levels of SOCS3 expression in maturing myeloid cells in bone marrow, it is not possible to determine whether there is rapid induction of neutrophil SOCS3 expression at sites of inflammation following their recruitment to these sites or whether neutrophils that constitutively express SOCS3 are recruited to the inflamed site. Our striking observation of the significant accentuation of SOCS3 expression in the centre of granulomas compared with the peripheral parts. It is likely that there is both a higher level of cytokines, such as IL-6, and of microbial products at the centre of the granuloma, causing increased levels of SOCS3 induction via both cytokine receptors and TLRs. The relatively short half-life of SOCS3 would mean that its expression relied on repeated or continuous induction, mirroring local cytokine or microbial product levels. Following IL-6 stimulation of myeloid cell lines, mRNA from SOCS-3 is detectable within $20 \mathrm{~min}$,

Fig. 5 SOCS3 expression in inflamed human tissues. All panels show double immunostaining with SOCS3 detection by the peroxidase technique (brown) and CD68 detection by the alkaline phosphatase technique $(r e d)$. a. Acute inflammation in acute appendicitis shows moderate to strong SOCS3 immunopositivity in $\mathrm{CD}^{+} 8^{+}$cells (arrows) and strong SOCS3 immunopositivity in a range of other leucocytes, particularly neutrophils (arrowheads). b In the colon in ulcerative colitis, high levels of nuclear and cytoplasmic SOCS3 immunopositivity were seen in neutrophils in crypt abscesses (arrowheads), as well as in epithelium adjacent to areas of acute inflammation (EP). A small focus of surface ulceration is shown and the underlying macrophages/dendritic cells (MAC) show strong SOCS3 expression. c Submucosal blood vessel in Crohn's disease containing abundant $\mathrm{SOCS}^{+}$neutrophils (arrows) and showing strong endothelial SOCS3 expression, with adjacent $\mathrm{SOCS}^{+}$macrophages/dendritic cells (arrowheads). d Giant cell (arrow) from the submucosa in colonic Crohn's disease, showing strong nuclear and weaker cytoplasmic SOCS3 immunopositivity, together with macrophages/dendritic cells (arrowheads) showing similar SOCS3 expression. e Non-necrotising granulomas of sarcoidosis (one granuloma is circled) in which macrophages show moderate nuclear and cytoplasmic SOCS3 immunopositivity with marked accentuation in the centre of the granuloma (arrow). f Granuloma in temporal arteritis showing similar features to e. All scale bars (bottom right of each panel) $50 \mathrm{~m}$. (Color figure online) 

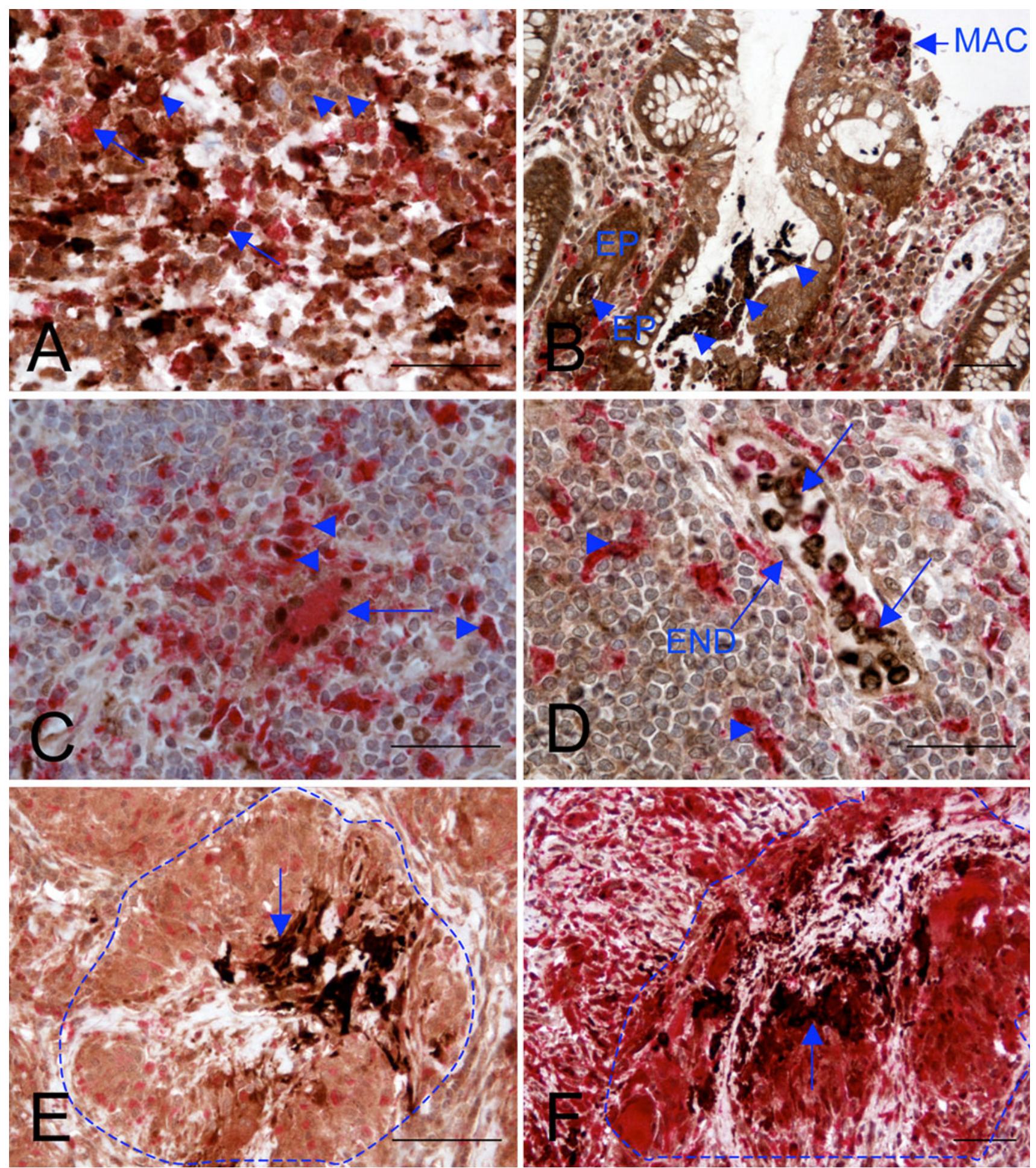

declining to basal levels after $8 \mathrm{~h}$ (Larsen and Ropke 2002). Our observation of more intense immunostaining, suggesting higher SOCS3 expression levels, in acute than chronic or granulomatous inflammation is also consistent with the time course of SOCS3 induction, as well as possibly being related to higher cytokine levels in acute inflammation.
In summary, we have demonstrated both cytoplasmic and nuclear localisation of the regulatory protein SOCS3 and have performed the first detailed expression study on SOCS3 by means of immunohistochemistry on human tissues. Commensurate with its induction by a range of cytokines, with a rapid time course of induction and subsequent rapid downregulation, a very significant increase in 
Table 2 Inflammatory conditions, their normal counterparts and changes in percentage of $\mathrm{CD}^{+} 8^{+}$cells expressing SOCS3 in various inflammatory conditions

\begin{tabular}{|c|c|c|c|c|c|}
\hline Inflammatory Condition & $\begin{array}{l}\text { Mean } \% \mathrm{CD}^{+} 8^{+} \\
\text {cells expressing } \\
\text { SOCS3 (counted } \\
\text { in } 4 \text { fields) }(\%)\end{array}$ & $\begin{array}{l}\text { Type of } \\
\text { Inflammation }\end{array}$ & $\begin{array}{l}\text { Corresponding } \\
\text { Normal Tissue }\end{array}$ & $\begin{array}{l}\text { Mean } \% \mathrm{CD}^{+} 8^{+} \\
\text {cells expressing } \\
\text { SOCS3 } \\
\text { (counted in } 4 \\
\text { fields) }(\%)\end{array}$ & $\begin{array}{l}\% \mathrm{CD}^{2} 8^{+} \text {cells expressing } \\
\text { SOCS3 in inflamed } \\
\text { compared with } \\
\text { normal tissue }\end{array}$ \\
\hline Lymph node sarcoidosis & 62.35 & Granulomatous & Normal lymph node & 27.42 & $\uparrow p=0.0014$ \\
\hline Crohn's Disease & 43.10 & Granulomatous & Normal colon & 11.04 & $\uparrow p<0.0001$ \\
\hline $\mathrm{UC}$ & 40.03 & Acute-on-chronic & Normal colon & 11.04 & $\uparrow p<0.0001$ \\
\hline Acute appendicitis & 32.69 & Acute & Normal colon & 11.04 & $\uparrow p=0.0020$ \\
\hline Hidradenitis suppurativa & 24.60 & Granulomatous & Normal skin & 18.81 & $\nearrow p=0.1572$ \\
\hline Radicular cyst & 40.65 & Acute-on-chronic & Benign fibroepithelial polyp & 34.33 & $\nearrow p=0.3123$ \\
\hline Temporal arteritis & 53.29 & Granulomatous & Normal temporal artery & 31.46 & $\uparrow p=0.0239$ \\
\hline
\end{tabular}

expression at sites of inflammation, particularly in areas where the inflammation is acute and/or cytokines are present at highest levels. This makes SOCS3 a potential marker of sites at which there are significant levels of cytokines and it may be a more stable and useful marker of ongoing inflammation than the cytokines themselves.

Acknowledgments Work in the laboratory of DRG is funded by the British Heart Foundation. MA was the recipient of a Wellcome Trust Cardiovascular Initiative PhD studentship. ES is supported by a grant from the Oxford University Medical Research Fund.

Conflicts of interest The authors have no conflicts of interest to declare.

Open Access This article is distributed under the terms of the Creative Commons Attribution Noncommercial License which permits any noncommercial use, distribution, and reproduction in any medium, provided the original author(s) and source are credited.

\section{References}

Berlato C, Cassatella MA, Kinjyo I, Gatto L, Yoshimura A, Bazzoni F (2002) Involvement of suppressor of cytokine signaling-3 as a mediator of the inhibitory effects of il-10 on lipopolysaccharideinduced macrophage activation. J Immunol 168(12):6404-6411

Chen Z, Laurence A, Kanno Y, Pacher-Zavisin M, Zhu BM, Tato C, Yoshimura A, Hennighausen L, O'Shea JJ (2006) Selective regulatory function of socs 3 in the formation of il-17-secreting $t$ cells. Proc Natl Acad Sci U S A 103(21):8137-8142. doi:10.1073/ pnas.0600666103

Croker BA, Krebs DL, Zhang JG, Wormald S, Willson TA, Stanley EG, Robb L, Greenhalgh CJ, Forster I, Clausen BE, Nicola NA, Metcalf D, Hilton DJ, Roberts AW, Alexander WS (2003) Socs3 negatively regulates il-6 signaling in vivo. Nat Immunol 4(6):540-545. doi:10.1038/ni931

Croker BA, Metcalf D, Robb L, Wei W, Mifsud S, DiRago L, Cluse LA, Sutherland KD, Hartley L, Williams E, Zhang JG, Hilton DJ, Nicola NA, Alexander WS, Roberts AW (2004) Socs3 is a critical physiological negative regulator of g-csf signalling and emergency granulopoiesis. Immunity 20(2):153-165
Croker BA, Kiu H, Nicholson SE (2008) Socs regulation of the jak/ stat signalling pathway. Semin Cell Dev Biol 19(4):414-422. doi:10.1016/j.semcdb.2008.07.010

Dalpke A, Heeg K, Bartz H, Baetz A (2008) Regulation of innate immunity by suppressor of cytokine signaling (socs) proteins. Immunobiology 213(3-4):225-235. doi:10.1016/j.imbio.2007. 10.008

Dimitriou ID, Clemenza L, Scotter AJ, Chen G, Guerra FM, Rottapel R (2008) Putting out the fire: Coordinated suppression of the innate and adaptive immune systems by socs1 and socs 3 proteins. Immunol Rev 224:265-283. doi:10.1111/j.1600-065X. 2008.00659.x

Ding Y, Chen D, Tarcsafalvi A, Su R, Qin L, Bromberg JS (2003) Suppressor of cytokine signaling 1 inhibits il-10-mediated immune responses. J Immunol 170(3):1383-1391

Donnelly RP, Dickensheets H, Finbloom DS (1999) The interleukin-10 signal transduction pathway and regulation of gene expression in mononuclear phagocytes. J Interferon Cytokine Res 19(6):563-573. doi:10.1089/107999099313695

Elliott J, Suessmuth Y, Scott LM, Nahlik K, McMullin MF, Constantinescu SN, Green AR, Johnston JA (2009) Socs3 tyrosine phosphorylation as a potential bio-marker for myeloproliferative neoplasms associated with mutant jak2 kinases. Haematologica 94(4):576-580. doi:10.3324/haematol.2008.002 352

Feldmann M, Maini RN (2003) Lasker clinical medical research award. Tnf defined as a therapeutic target for rheumatoid arthritis and other autoimmune diseases. Nat Med 9(10):1245-1250. doi:10.1038/nm939

Fritz JH, Le Bourhis L, Magalhaes JG, Philpott DJ (2008) Innate immune recognition at the epithelial barrier drives adaptive immunity: Apcs take the back seat. Trends Immunol 29(1): 41-49. doi:10.1016/j.it.2007.10.002

Hart DN (1997) Dendritic cells: unique leukocyte populations which control the primary immune response. Blood 90(9):3245-3287

Hookham MB, Elliott J, Suessmuth Y, Staerk J, Ward AC, Vainchenker W, Percy MJ, McMullin MF, Constantinescu SN, Johnston JA (2007) The myeloproliferative disorder-associated jak2 v617f mutant escapes negative regulation by suppressor of cytokine signaling 3. Blood 109(11):4924-4929. doi:10.1182/blood-200608-039735

Imai K, Kurita-Ochiai T, Ochiai K (2003) Mycobacterium bovis bacillus calmette-guerin infection promotes socs induction and inhibits ifn-gamma-stimulated jak/stat signaling in j774 macrophages. FEMS Immunol Med Microbiol 39(2):173-180 
Isomaki P, Alanara T, Isohanni P, Lagerstedt A, Korpela M, Moilanen T, Visakorpi T, Silvennoinen O (2007) The expression of socs is altered in rheumatoid arthritis. Rheumatology (Oxford) 46(10):1538-1546. doi:10.1093/rheumatology/kem198

Kimura A, Kinjyo I, Matsumura Y, Mori H, Mashima R, Harada M, Chien KR, Yasukawa H, Yoshimura A (2004) Socs3 is a physiological negative regulator for granulopoiesis and granulocyte colony-stimulating factor receptor signaling. J Biol Chem 279(8):6905-6910. doi:10.1074/jbc.C300496200

Larsen L, Ropke C (2002) Suppressors of cytokine signalling: Socs. APMIS 110(12):833-844

Le Y, Zhu BM, Harley B, Park SY, Kobayashi T, Manis JP, Luo HR, Yoshimura A, Hennighausen L, Silberstein LE (2007) Socs3 protein developmentally regulates the chemokine receptor cxcr4fak signaling pathway during $\beta$ lymphopoiesis. Immunity 27(5):811-823. doi:10.1016/j.immuni.2007.09.011

Lee KH, Moon KJ, Kim HS, Yoo BC, Park S, Lee H, Kwon S, Lee ES, Yoon S (2008) Increased cytoplasmic levels of cis, socs1, socs2, or socs3 are required for nuclear translocation. FEBS Lett 582(15):2319-2324. doi:10.1016/j.febslet.2008.05.039

Li Y, Chu N, Rostami A, Zhang GX (2006) Dendritic cells transduced with socs-3 exhibit a tolerogenic/dc2 phenotype that directs type $2 \mathrm{TH}$ cell differentiation in vitro and in vivo. J Immunol 177(3): $1679-1688$

Li Y, de Haar C, Chen M, Deuring J, Gerrits MM, Smits R, Xia B, Kuipers EJ, van der Woude CJ (2010) Disease-related expression of the il6/stat3/socs 3 signalling pathway in ulcerative colitis and ulcerative colitis-related carcinogenesis. Gut 59(2):227-235. doi:10.1136/gut.2009.184176

Liddiard K, Welch JS, Lozach J, Heinz S, Glass CK, Greaves DR (2006) Interleukin-4 induction of the cc chemokine tarc (ccl17) in murine macrophages is mediated by multiple stat6 sites in the tarc gene promoter. BMC Mol Biol 7:45. doi:10.1186/14712199-7-45

Lin WW, Karin M (2007) A cytokine-mediated link between innate immunity, inflammation, and cancer. J Clin Invest 117(5): 1175-1183. doi:10.1172/JCI31537

Liu X, Zhang Y, Yu Y, Yang X, Cao X (2008a) Socs3 promotes tlr4 response in macrophages by feedback inhibiting tgf-beta1/smad3 signaling. Mol Immunol 45(5):1405-1413. doi:10.1016/ j.molimm.2007.08.018

Liu Y, Stewart KN, Bishop E, Marek CJ, Kluth DC, Rees AJ, Wilson HM (2008b) Unique expression of suppressor of cytokine signaling 3 is essential for classical macrophage activation in rodents in vitro and in vivo. J Immunol 180(9):6270-6278

Marine JC, McKay C, Wang D, Topham DJ, Parganas E, Nakajima H, Pendeville H, Yasukawa H, Sasaki A, Yoshimura A, Ihle JN
(1999) Socs3 is essential in the regulation of fetal liver erythropoiesis. Cell 98(5):617-627

Nathan C (2002) Points of control in inflammation. Nature 420(6917):846-852. doi:10.1038/nature01320

O'Shea JJ, Murray PJ (2008) Cytokine signaling modules in inflammatory responses. Immunity 28(4):477-487. doi:10.1016/ j.immuni.2008.03.002

Rakshit DS, Ly K, Sengupta TK, Nestor BJ, Sculco TP, Ivashkiv LB, Purdue PE (2006) Wear debris inhibition of anti-osteoclastogenic signaling by interleukin-6 and interferon-gamma Mechanistic insights and implications for periprosthetic osteolysis. J Bone Joint Surg Am 88(4):788-799. doi:10.2106/JBJS. E.00711

Roberts AW, Robb L, Rakar S, Hartley L, Cluse L, Nicola NA, Metcalf D, Hilton DJ, Alexander WS (2001) Placental defects and embryonic lethality in mice lacking suppressor of cytokine signaling 3. Proc Natl Acad Sci U S A 98(16):9324-9329. doi:10.1073/pnas. 161271798

Soilleux EJ, Morris LS, Rushbrook S, Lee B, Coleman N (2002) Expression of human immunodeficiency virus (hiv)-binding lectin dc-signr: consequences for hiv infection and immunity. Hum Pathol 33(6):652-659

Starr R, Willson TA, Viney EM, Murray LJ, Rayner JR, Jenkins BJ, Gonda TJ, Alexander WS, Metcalf D, Nicola NA, Hilton DJ (1997) A family of cytokine-inducible inhibitors of signalling. Nature 387(6636):917-921

Wang YC, He F, Feng F, Liu XW, Dong GY, Qin HY, Hu XB, Zheng MH, Liang L, Feng L, Liang YM, Han H (2010) Notch signaling determines the $m 1$ versus $m 2$ polarization of macrophages in antitumor immune responses. Cancer Res 70(12):4840-4849. doi:10.1158/0008-5472.CAN-10-0269

Wong PK, Egan PJ, Croker BA, O’Donnell K, Sims NA, Drake S, Kiu H, McManus EJ, Alexander WS, Roberts AW, Wicks IP (2006) Socs-3 negatively regulates innate and adaptive immune mechanisms in acute il-1-dependent inflammatory arthritis. J Clin Invest 116(6):1571-1581. doi:10.1172/JCI25660

Xu Y, Kershaw NJ, Luo CS, Soo P, Pocock MJ, Czabotar PE, Hilton DJ, Nicola NA, Garrett TP, Zhang JG (2010) Crystal structure of the entire ectodomain of gp130: Insights into the molecular assembly of the tall cytokine receptor complexes. J Biol Chem. doi:10.1074/jbc.C110.129502

Yoshimura A, Naka T, Kubo M (2007) Socs proteins, cytokine signalling and immune regulation. Nat Rev Immunol 7(6): 454-465. doi:10.1038/nri2093 